\title{
Análisis de las características de la Realidad Aumentada aplicada a la educación
}

\section{Analysis of the characteristics of Augmented Reality applied to education}

\author{
Quezada Sarmiento Ramiro Hernán ${ }^{1}$ \\ https://orcid.org/0000-0002-9552-8486 \\ Universidad Técnica de Machala, Ecuador \\ Rivera Escriba Luis Antonio ${ }^{2}$ \\ https://orcid.org/0000-0002-5029-2561 \\ Universidad Estatal del Norte Fluminense, Río de Janeiro, Brasil \\ Loján Cueva Edison Luis ${ }^{3}$ \\ https://orcid.org/0000-0002-7092-1281 \\ Loja Mora Nancy Magaly ${ }^{4}$ \\ https://orcid.org/0000-0002-5583-4278 \\ Universidad Técnica de Machala, Ecuador
}

Recibido:20-09-2020

Aceptado: 22-12-2020

\section{Cita Recomendada}

Quezada, R., Rivera, L., Loján, L. \& Lona, N. (2020). Análisis de las características de la Realidad Aumentada aplicada a la educación. Hamut'ay, 7 (3), 75-85

http://dx.doi.org/10.21503/hamu.v7i3.2202

\section{Resumen}

El avance de las tecnologías de información y comunicación y la incorporación de tecnologías emergentes en la educación, ha dado lugar a al desarrollo de herramientas innovadoras como es el caso de las aplicaciones de Realidad Aumentada, en las cuales se combinan elementos virtuales en ambientes reales al mismo tiempo. Estas aplicaciones vienen siendo utilizadas en diferentes áreas educativas, con mayor frecuencia en temas con contenidos abstractos, con la intención de facilitar la asimilación de los mismos. Varias de éstas aplicaciones de realidad aumentada fueron analizadas en la presente investigación, con el objetivo de determinar sus características y de esta manera sirvan de guía para futuras aplicaciones.

Palabras clave: Realidad aumentada, realidad aumentada en educación, aplicaciones de realidad aumentada.

\footnotetext{
1. Ingeniero en Sistemas Informáticos y Computación en la Universidad Técnica Particular de Loja. Docente Investigador de la Universidad Técnica de Machala. Estudiante de Doctorado en Ingeniería de Sistemas e Informática en la Universidad Nacional Mayor de San Marcos Lima-Perú. rquezada@ utmachala.edu.ec

2. Posee graduación en Ciencia de la Computación por la Universidad Nacional Mayor de San Marcos, Maestría y Doctorado en Ciencia de la Computación por la Pontificia Universidad Católica de Rio de Janeiro. Actualmente es Investigador Asociado de la Universidad Estatal del Norte Fluminense Río de Janeiro-Brasil. arivera@uenf.br

3. Ingeniero de Sistemas por la Universidad Católica de Cuenca, Especialista en Docencia Universitaria por la Universidad Católica de Cuenca, Magíster en Sistemas de Información Gerencial por la Universidad Católica de Cuenca - ESPOL, Docente Titular de la Universidad Técnica de Machala. elojan@utmachala.edu.ec

4. Ingeniero en Sistemas Informáticos y Computación por la UTPL, Magister en Docencia y Gerencia en Educación Superior por la Universidad de Guayaquil, Magíster en Gestión Estratégica de las Tecnologías de la Información por la Universidad de Cuenca.nmloja@utmachala.edu.ec
} 


\begin{abstract}
The advancement of information and communication technologies and the incorporation of emerging technologies in education, has led to the development of innovative tools such as Augmented Reality applications, in which virtual elements are combined in real environments at the same time. These applications have been used in different educational areas, more frequently in subjects with abstract content, with the intention of facilitating their assimilation. Several of these augmented reality applications were analyzed in this research, in order to determine their characteristics and thus serve as a guide for future applications.
\end{abstract}

Keywords: Augmented reality, augmented reality in education, augmented reality applications.

\section{Introducción}

La difícil tarea de utilizar e incorporar herramientas tecnológicas dentro del ámbito educativo, tiene como una de sus principales causas, la carencia de investigaciones sobre tecnologías emergentes en éstos ámbitos. Con el surgimiento de la Realidad Aumentada (RA), investigadores y educadores se han visto atraídos por alternativas tecnológicas interesantes e innovadoras que permiten crear herramientas de enseñanza-aprendizaje para beneficio de la educación. La RA es una tecnología que proporciona una interfaz interactiva única, en la que se combinan elementos virtuales y reales y, donde el usuario puede trabajar e interactuar simultáneamente con el mundo real y los objetos virtuales de forma natural.

En la actualidad la RA se ha vuelto más accesible, puesto que no requiere el uso de equipo especializado y puede ser fácilmente utilizado en dispositivos móviles; aunque, por otro lado, investigaciones sobre las aplicaciones de RA en la educación todavía se encuentra en una etapa temprana, existiendo una falta de investigación sobre los efectos e implicaciones de la RA en este ámbito (Akçayır \& Akçayır, 2017; Wu, H. et al., 2013). Varias de las aplicaciones de RA que han sido probadas y son utilizadas como herramientas de apoyo en el proceso de enseñanza-aprendizaje, han tenido resultados positivos y otras que no lograron los efectos deseados; por lo que, la presente investigación tiene como propósito indagar entre las diferentes aplicaciones de RA, con el objetivo de determinar las características que deberían tener futuras aplicaciones de RA enfocadas al ámbito educativo, contribuyendo de esta manera con mayores hallazgos empíricos que be- neficien y mejoren el aprendizaje académico. Entre las aplicaciones consideradas dentro de la presente investigación, se encuentran: Elements 4D, Zookazam y Anatomy 4D, las cuales vienen siendo utilizadas en diferentes proyectos educativos en los que se obtuvieron resultados positivos. Adicionalmente se analizan dos investigaciones con experiencias en RA educativa que han servido para complementar el análisis correspondiente.

\section{Método}

Al tratarse de un estudio documental, la revisión de la literatura se basó en artículos científicos que envuelven al tema en cuestión. Como motor de búsqueda de la revisión se utilizó Google académico, de donde se obtuvieron algunas fuentes científicas relevantes con frases como: "realidad aumentada", "realidad aumentada en la educación", "aplicaciones de realidad aumentada en la educación" y algunas de sus variantes. Las fuentes científicas utilizadas, en su mayoría son del año 2015 hacia adelante y, algunas fuentes anteriores a 2015 por considerarse relevantes y necesarias para referir conceptos y enfoques dentro del estudio.

\section{Realidad Aumentada}

La realidad aumentada está descrita como una tecnología que combina objetos virtuales bidimensionales o tridimensionales, dentro de un entorno tridimensional real y proyectado en tiempo real. (Gede et al., 2015). Para Akçayir et al., (2016), un sistema de RA es caracterizado por la combinación de objetos virtuales y reales, per- 
fectamente alineados en un escenario real, en el que una persona pueda interactuar con ellos en tiempo real. Esta característica convierte a la RA en una tecnología inmersiva (Marín \& Sampedro-Rquena, 2019), especialmente en contextos educativos. En general, un sistema de realidad virtual (RV) y realidad aumentada (RA) fundamentalmente usa dispositivos de entrada y salida con posición y orientación espacial, que puede ser rastreada en una plataforma de computación con procesador gráfico apropiado, en base de un framework de manipulación de software de RV/ RA de entrada/salida y comportamiento de aplicaciones (Mossel et al., 2012), como se muestra en la Figura 1. Los datos capturados son llevados a la plataforma de computación y entregados a la RV/RA para que sean transformados y entregados como datos de entrada a la aplicación.

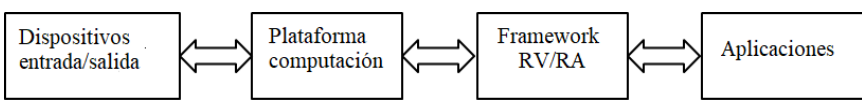

Figura.1. Framework de realidad virtual / realidad aumentada. Fuente: Elaboración propia (2020)

Esa conexión de dominios físico y digital permite que el humano pueda construir visiones completas de casos y fenómenos, como si todo fuese real. Las interacciones son realizadas a través de computadoras de escritorio con cámaras y, últimamente con mayor frecuencia, a través de móviles (Akcayir \& Akcayir, 2017). En los últimos años, la tendencia de los investigadores es la preferencia por el uso de aplicaciones móviles RA como herramientas educativas en sus diferentes niveles, con un 61\% (Ruiz-ariza et al., 2017); aplicaciones RA de escritorio con un $24 \%$ y otros (Kinect, HMD, 3D visión glasses, entre otros) con un 16\% (Akçayır \& Akçayır, 2017). Esto se debe a que el móvil es un dispositivo portátil que los estudiantes pueden utilizar para aplicaciones RA basadas en localización y por su disponibilidad en diversos entornos (Steinkuehler \& Squire, 2014), para una gran variedad de personas (Nincarean et al., 2013; Cheng \& Tsai, 2013).

La RA viene siendo utilizada en diferentes ámbitos como la Química (Nechypurenko et al., 2018; Habig, 2020; Irwansyah et al., 2018), Arquitectura (De La Fuente Prieto et al., 2017; Abdullah et al., 2017), Ingeniería (Wr et al., 2017; Li et al., 2017), Medicina (Andersen et al., 2016; (Kilgus et al., 2015), Marketing (Rauschnabel et al., 2019; Wedel et al., 2020; Scholz \& Duffy, 2018), entre otros.

Las diferentes aplicaciones de RA utilizan mecanismos que definen el grado de complejidad en los que se desenvuelven estos sistemas; funcionan como activadores de información y se encuentran clasificados en lo que se conoce como niveles de RA. Para Prendes, (2015), los niveles de RA se clasifican de la siguiente forma: Nivel 0, utiliza códigos de barra, códigos QR o reconocimiento de imágenes aleatorias y sirven como hiperenlaces a otros contenidos. Nivel 1, RA en la que se implementan marcadores, reconociendo patrones 2D y objetos 3D. Nivel 2, el reconocimiento lo realiza a través de GPS (Marín \& Sampedro-Rquena, 2019), brújula y en algunas ocasiones acelerómetros, evitando de esta manera el uso de marcadores. Nivel 3, visión aumentada en donde se emplean dispositivos como lentes de contacto de alta tecnología, Google Glass, entre otros.

\section{Realidad Aumentada en la educación}

Mediante la utilización de aplicaciones de RA se puede percibir beneficios en el proceso de enseñanza-aprendizaje y desarrollar competencias tecnológicas tanto de alumnos como de docentes (Cabero Almenara \& Barroso Osuna, 2016; Toledo \& Sánchez, 2017)

Entre los diversos estudios realizados sobre RA en educación (Akçayir et al., 2016), es evidente que la RA ofrece a la educación aspectos positivos que podrían mejorar significativamente los procesos educativos, promoviendo actitudes positivas en los estudiantes (Siwawetkul \& Koraneekij, 2018; Akçayir et al,2016; Marín \& Sampedro-Rquena, 2019). Se posibilita a los estudiantes a experimentar con situaciones y fenómenos científicos que no son posibles de realizar en el mundo real, situaciones que en ciertos casos no se pueden realizar por los elevados costos del equipamiento, disponibilidad física y de tiempo 
de las instalaciones; experimentos complejos y peligrosos que no se pueden realizar por las lesiones que pueden causar si existiera un error, experimentaciones en las que se necesitan largos periodos de tiempo para su ejecución, pero que esos tiempos pueden verse simplificados con la ayuda de aplicaciones RA. Un ejemplo claro es la investigación presentada por Chiu et al., (2015), en la que se desarrolló una aplicación que permite realizar procesos en un laboratorio de RA para análisis de fenómenos químicos de gas con visualizaciones a nivel molecular, aplicaciones como ésta facilitan la experimentación y una mejor comprensión (Cai et al., 2014b). El estudio de (Marín \& Sampedro-Rquena, 2019), menciona que la RA puede enriquecer textos planos como libros y apuntes, incorporando recursos interactivos. Para Cai et al. (2014a) la RA es más aplicable en los siguientes dos casos: 1) Cuando un fenómeno no se puede simular en la realidad y 2) Cuando los experimentos presentan problemas evidentes.

Constantemente se buscan formas de mejorar el aprendizaje en todos sus niveles, aún existe un grande porcentaje de estudiantes, principalmente de la educación básica, que no asimilan bien los conceptos de las materias, sobre todo de conceptos abstractos como las matemáticas, la química, la física, y otras (Oviatt et al., 2016.); como también materias de tecnologías e ingenierías (Lindgren et al., 2016), los conceptos abstractos de las ciencias pueden ser analizados por los estudiantes a nivel de objetos conjuntamente con sus propiedades, para un entendimiento efectivo y así aumentar la retención de conocimiento por experimentación (Billinghurst \& Dunser, 2012), orientar el análisis y síntesis de conceptos a través de visualizaciones 2D y 3D (Salinas et al., 2013), aprender conceptos matemáticos con RA (Bujak et al., 2013) . Con esta tecnología, los estudiantes fortalecen aspectos motivacionales (Spector et al., 2014; Khan, T. et al., 2019), motivación que les permite aprender y mejorar sus prácticas en la educación, como es el caso del trabajo presentado por Liu et al. (2016), juego de RA móvil local de aprendizaje de lenguas; algunas aplicaciones de
RA se combinan con juegos serios para el aprendizaje (Moloney et al., 2017), y algunas otras aprovechan la atracción que los juegos producen para inducir el conocimiento en los estudiantes (Tomi \& Rambli, 2013; Rambli et al., 2013). Por el contrario, la falta de motivación puede ser una causa del bajo interés en la ciencia por parte de los niños (Laine et al., 2016).

\section{Aplicaciones AR enfocadas a la educación}

Dentro de la presente investigación se consideró tres aplicaciones que forman parte de varios estudios y proyectos educativos, obteniendo resultados positivos en la educación y, en muchos casos pueden emplearse como complemento a los métodos de aprendizaje tradicionales (Sampaio \& Almeida, 2016); de igual forma se consideraron dos experiencias educativas con RA que permiten complementar y evidenciar en conjunto características importantes que se debería considerar en futuras aplicaciones con RA.

\section{Elements 4D}

Consiste en una aplicación de realidad aumentada móvil que, para su funcionamiento utiliza marcadores en forma de cubos que pueden descargarse conjuntamente con la aplicación; éstos marcadores contienen distintos elementos de la tabla periódica, los cuales al ser escaneados mediante la aplicación en un dispositivo móvil con sistema operativo iOS o Android, permiten visualizar el elemento químico con sus características e interactuar a través de un modelo para conseguir combinaciones de elementos, entre otras opciones. Ver Figura 2.

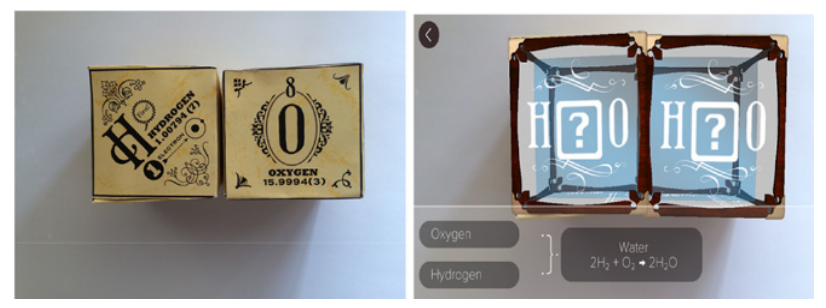

Figure 2. Ilustración de la reacción química entre hidrógeno $(\mathrm{H})$ y oxígeno $(\mathrm{O})$ en Elementos 4D.

Fuente: (Yang et al., 2018) 
Para Yang et al., (2018), utilizar la aplicación permitió explorar la percepción de varios profesores de química sobre contenidos químicos; dentro de la investigación participaron quince profesores de química en formación en una Universidad de China, con la finalidad de que pudieran interactuar con la aplicación durante dos semanas y de esta manera poder determinar mediante entrevistas sobre su uso, percepción y experiencia con la aplicación en contenidos químicos. Los resultados mostraron como aspectos positivos que, la mayoría $(n=12)$, al tener una interacción directa visible con los elementos, tuvieron una mejor comprensión de los elementos y sus propiedades y, por ende, representa un refuerzo en los contenidos del curso. Por otro lado, algunos participantes $(n=5)$, mencionaron que la aplicación puede ayudar a disminuir el temor de manipular reacciones químicas peligrosas en los laboratorios, puesto que, la aplicación permite observar una reacción de este tipo en un entorno seguro, algo similar al trabajo presentado por Dunleavy \& Dede, (2014). Algunos participantes $(n=10)$, mencionaron que la aplicación podría ser una gran alternativa para la educación química en centros rurales, en donde no se cuenta con laboratorios, productos o equipos. La mayoría de participantes sugieren la provisión de lecciones dentro de la aplicación, lo que permitiría una rápida adaptación a la aplicación y de esta manera llevar a cabo actividades de enseñanza-aprendizaje más relevantes.

Entre las limitaciones que se mencionan, se encuentra la falta de contenidos profesionales, por lo que la aplicación serviría para un nivel de educación medio y no superior. De igual forma se menciona la falta de efectos visuales durante el proceso de reacción química, el número limitado de elementos químicos con los que cuenta actualmente la aplicación y algunos problemas técnicos que formaron parte de la experiencia de usuario negativa.

\section{Zookazam}

Esta aplicación está dentro del grupo de aplicaciones RA para móviles, orientada al ámbito edu- cativo y, permite visualizar cerca de 45 animales e interactuar con la aplicación de manera prácticamente realista, dando la oportunidad a sus usuarios de conocer más acerca del mundo animal. Figura 3 muestra un ejemplo de la realidad aumentada generada por la aplicación.

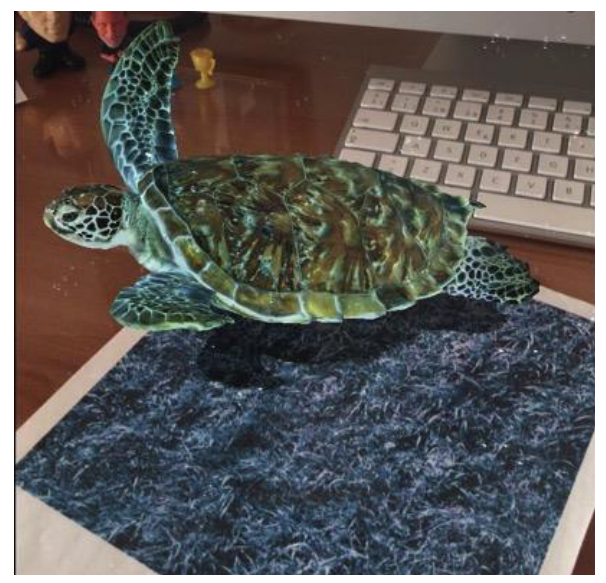

Figura 3: Una de las imágenes generadas por ZooKazam Fuente: (Martínez et al., 2017)

Es una aplicación que utiliza marcadores que pueden descargarse de su página web http:// www.zookazam.com/, aunque actualmente la aplicación permite al usuario crear sus propios marcadores usando la cámara de sus dispositivo. La visualización es variada dependiendo del tipo de modelo seleccionado dentro de la aplicación, sumándose a cada visualización la oportunidad de seleccionar comandos que permiten escuchar características y costumbres del animal elegido. Ver Figura 4.

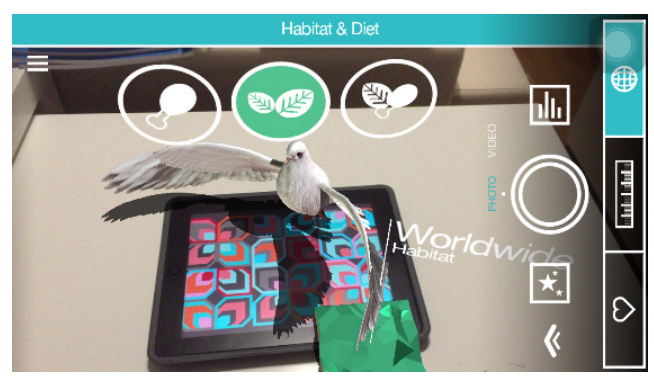

Figura 4: Características adicionales dentro de la aplicación ZooKazam.

Fuente: http://www.enlanubetic.com.es/2015/12/zookazam.html

Según (Blas Padilla et al., 2019), en su estudio sobre el uso de aplicaciones RA en el ámbito educativo, la aplicación Zookazam es una de las más valoradas por los estudiantes, debido a la facilidad de manejo que con la que cuenta y a su 
aplicabilidad en contextos infantiles colectivos, lo que la convierte en una herramienta que aporta al proceso formativo. Para (Vázquez-Cano et al., 2020), representa una herramienta muy importante en términos de usabilidad y facilidad de uso, especialmente para aquellos estudiantes de educación primaria, en la adquisición de nuevos conocimientos.

\section{Anatomy 4D}

Aplicación móvil que emplea RA y que tiene como propósito permitir a los usuarios que interactúen con imágenes del cuerpo humano. Representa una buena oportunidad para que los estudiantes puedan observar e interactuar de manera innovadora y atractiva con partes como el esqueleto, músculos, órganos y sistemas del cuerpo humano; además, la aplicación permite la experimentación con modelos masculino y femenino para un estudio diferenciado. Para su funcionamiento la aplicación utiliza una imagen de destino y la cámara de un dispositivo móvil para mostrar la imagen objetivo. Ver Figura 5.

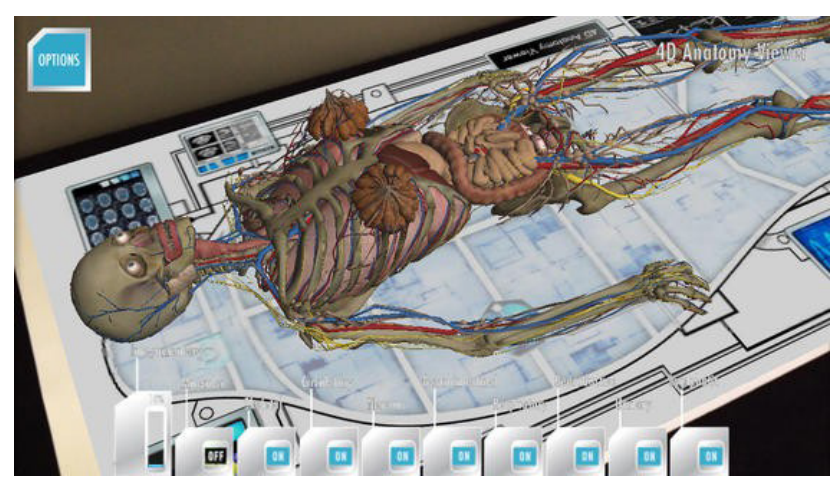

Figura 5: Imagen de uno de los modelos generados por Anatomy 4D

Fuente: https://edshelf.com/tool/anatomy-4d/

La imagen de la anatomía puede girar $360^{\circ}$, ofreciendo un aspecto muy realista como por ejemplo la imagen del corazón, en la que además de sus partes los estudiantes pueden oír y ver los latidos del corazón. Ver Figura 6.

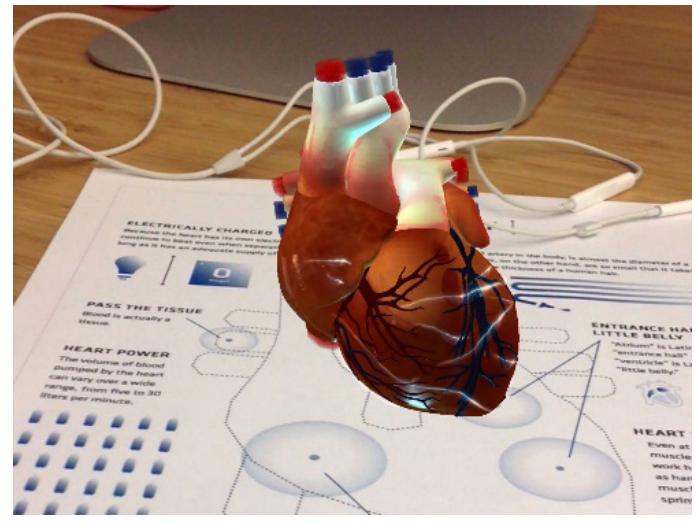

Figura 6: Aspecto realista del corazón.

Fuente: https://blog.library.tc.columbia.edu

Para (Khan, T. et al., 2019), luego de experimentar con aplicación Anatomy 4D como parte de su estudio, destacan que, el factor atención hacia los contenidos mejoró notablemente en comparación con las notas de anatomía convencionales, lo que da un inicio a la motivación de los estudiantes por aprender. Dentro del mismo estudio se resaltan otros factores que mejoraron notablemente como, la confianza y la satisfacción que sintieron los estudiantes luego de haber utilizado la aplicación, manifestando la seguridad de trabajar con ésta herramienta y el entretenimiento con el manejo de la misma.

Otras experiencias de Realidad Aumentada en la educación

En la investigación realizada por ( $\mathrm{Wu}, \mathrm{Y}$. et al., 2016), desarrollaron un juego de cartas interactivo con tecnología de realidad aumentada, como ayuda didáctica basada en juegos para impartir el contenido del curso de ciencias de la escuela primaria. La aplicación funciona en un dispositivo móvil con sistema Android y utiliza un conjunto de cartas imprimibles (marcadores), las cuales, al ser captadas por la cámara, presentan en pantalla el modelo tridimensional correspondiente. Ver Figura 7 a). 
Figura 7:
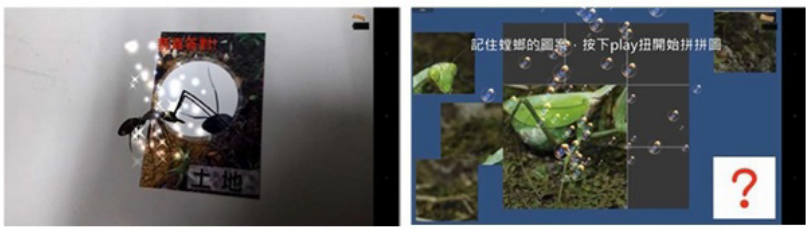

a) Imagen tridimensional de una carta.

Fuente: (Wu. Y. et al., 2016)

El objetivo del juego de cartas propuesto, los estudiantes pueden familiarizarse con apariencia, características y comportamiento de alimentación de los insectos; para lograrlo, la aplicación contiene juegos de rompecabezas y posteriormente un quiz que permite evidenciar el progreso de aprendizaje de los estudiantes. Ver Figura 7 b). La aplicación según Wu, Y. et al. (2016), constituye una herramienta motivadora que no requiere de la implementación de tecnología costosa, además de ser muy intuitiva y facilitar la interacción en los estudiantes de nivel educativo de primaria. Este estudio reafirma la efectividad que han tenido otros estudios relacionados con juegos educativos (Freitas, 2018; Nagalingam \& Ibrahim, 2015), y de algunos relacionados con juegos RA para el aprendizaje y la interacción social (Streitz \& Markopoulos, 2017).

Otra de las experiencias de realidad aumentada en la educación es la realizada por (Cai et al., 2014a); en esta investigación se diseñó una aplicación de computadora con realidad aumentada, seis marcadores impresos que son capturados por la cámara y un formulario de actividades. La aplicación cuenta con cuatro aplicaciones específicas de composición de sustancias químicas, las mismas que fueron utilizadas por un grupo de estudiantes de secundaria en China. Ver Figura 8

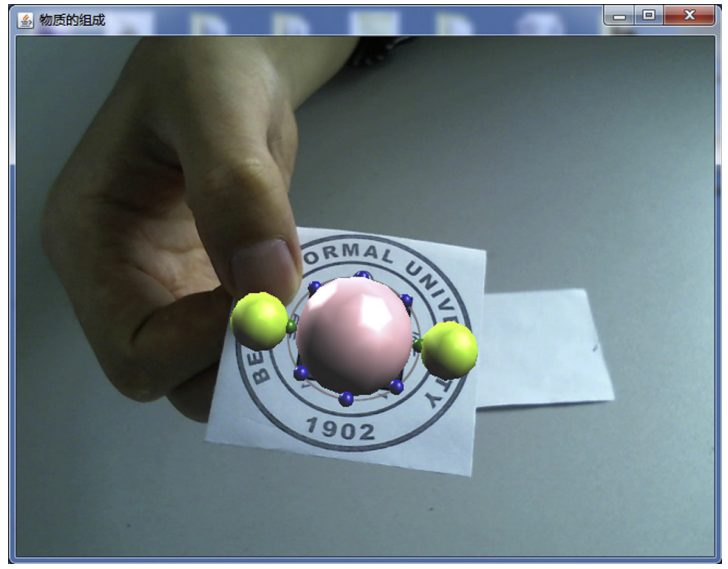

Figura 8: Representación de una sustancia química.

Fuente: (Cai et al., 2014a)

Durante la experimentación, los estudiantes que participaron de la misma, manipularon marcadores para controlar, combinar e interactuar con micro partículas reflejadas en un modelo 3D, situaciones que no son frecuentes en la vida real (Cai et al., 2014b), pero que ofrecen ventajas en comparación con laboratorios reales (Potkonjak et al., 2016). Para el funcionamiento de la aplicación se utilizaron marcadores que permitieron una serie de experimentos basados en la indagación. Los resultados obtenidos mostraron que esta herramienta de aprendizaje asistido por computadora, es más eficaz para estudiantes de bajo rendimiento académico. Otra de los aspectos a resaltar es la actitud positiva que los estudiantes mostraron con respecto al uso de la aplicación, fomentando además la cooperación en los grupos de trabajo.

\section{Conclusiones}

Es evidente que las aplicaciones consideradas en el estudio tienen como característica principal la motivación del aprendizaje en los estudiantes, permitiéndoles adquirir un grado superior de autonomía, adoptando de forma natural un rol activo y dinámico en la construcción de su propio aprendizaje. Otra característica que ofrece es la interactividad a través de sus imágenes tridimensionales, permitiendo enriquecer los materiales de texto plano con información adicional; el nivel de interacción dependerá de la complejidad de la tecnología incorporada y del usuario 
objetivo para el que esté diseñada.

La utilización de interfaces interactivas en las aplicaciones expuestas, dan como resultado una mejora en la atención, confianza y satisfacción en los estudiantes; por lo tanto, la interfaz de usuario en las aplicaciones constituye una características importante, puesto que sirve de guía y base para generar trabajo de manera intuitiva, de esta forma el uso de la aplicación no se convierte en una dificultad; Oviatt et al., (2016), observan que cuando incrementa el nivel de dificultad con la tecnología, hace que aumente el grado de dificultad para los estudiantes. Una de las características que sobresale en el estudio de Cai et al., (2014), es la cooperación en grupos de trabajo que permite la aplicación, logrando que los estudiantes puedan compartir ideas y despejar inquietudes mediante la experimentación con una interfaz inmersiva.

A través del trabajo propuesto por Wu, Y. et al. (2016), se puede entender que el desarrollo de aplicaciones que combinen RA con juegos alcanzan resultados favorables para el aprendizaje, en varias situaciones, propósitos y edades (Lester et al., 2014; Lucardie, 2014). Es también evidente que, gracias al avance tecnológico y al protagonismo de los dispositivos móviles (Akçayır \& Akçayır, 2017), la RA está cada vez más cerca de las personas, por lo que se debería aprovechar su uso para desarrollar el trabajo remoto y permitir que los estudiantes puedan aprender de forma más accesible.

Finalmente, es meritorio aclarar que las características obtenidas representan una primera guía para el diseño de aplicaciones de RA, dejando para futuras investigaciones temas importantes relacionados con contenidos pedagógicos, metodologías de aprendizaje que incentivan a los estudiantes a desarrollar sus conocimientos (Khan, A. et al., 2017), niveles de educación, entre otros.

\section{Referencias bibliográficas}

Abdullah, F., Kassim, M. H. Bin, \& Sanusi, A. N. Z. (2017). Go virtual: Exploring augmented reality application in representation of steel architectural construction for the enhancement of ar- chitecture education. Advanced Science Letters, 23(2), 804-808.

https://doi.org/10.1166/asl.2017.7449 Akçayir, M., Akçayir, G., Pektaş, H. M., \& Ocak, M. A. (2016). Augmented reality in science laboratories: The effects of augmented reality on university students' laboratory skills and attitudes toward science laboratories. Computers in Human Behavior, 57, 334-342. https://doi.org/10.1016/j.chb.2015.12.054

Akçayır, M., \& Akçayır, G. (2017). Advantages and challenges associated with augmented reality for education: A systematic review of the literature. Educational Research Review, 20, 1-11. https://doi.org/10.1016/j.edurev.2016.11.002 Andersen, D., Popescu, V., Cabrera, M. E., Shanghavi, A., Gomez, G., Marley, S., Mullis, B., \& Wachs, J. P. (2016). Medical telementoring using an augmented reality transparent display. Surgery (United States), 159(6), 1646-1653. https://doi.org/10.1016/j.surg.2015.12.016 Billinghurst, M., \& Dunser, A. (2012). Vocational Training Council Note : IEEE Computer Society, 56-63. https://doi.org/10.1109/MC.2012.111 Blas Padilla, D., Vázquez Cano, E., Morales Cevallos, M., \& López Meneses, E. (2019). Uso de apps de realidad aumentada en las aulas universitarias. Campus Virtuales : Revista Científica Iberoamericana de Tecnología Educativa, 8(1), 37-48.

Bujak, K. R., Radu, I., Catrambone, R., Macintyre, B., Zheng, R., \& Golubski, G. (2013). Computers \& Education A psychological perspective on augmented reality in the mathematics classroom. Computers \& Education, 68, 536-544.

https://doi.org/10.1016/j.compedu.2013.02.017 Cabero Almenara, J., \& Barroso Osuna, J. (2016). Posibilidades educativas de la Realidad Aumentada. Journal of New Approaches in Educational Research, 6(1), 44-50. https://doi.org/10.7821/ naer.2016.1.140

Cai, S., Wang, X., \& Chiang, F. (2014a). Computers in Human Behavior A case study of Augmented Reality simulation system application in a chemistry course. Computers in Human Behavior, 37, 31-40. https://doi.org/10.1016/j. chb.2014.04.018

Cai, S., Wang, X., \& Chiang, F. K. (2014b). A case 
study of Augmented Reality simulation system application in a chemistry course. Computers in Human Behavior, 37, 31-40. https://doi.org/10.1016/j.chb.2014.04.018

Cheng, K. H., \& Tsai, C. C. (2013). Affordances of Augmented Reality in Science Learning: Suggestions for Future Research. Journal of Science Education and Technology, 22(4), 449-462. https://doi.org/10.1007/s10956-012-9405-9

Chiu, J. L., Dejaegher, C. J., \& Chao, J. (2015). The effects of augmented virtual science laboratories on middle school students' understanding of gas properties. Computers and Education, 85, 59-73. https://doi.org/10.1016/j.compedu.2015.02.007 De La Fuente Prieto, J., Castaño Perea, E., \& Labrador Arroyo, F. (2017). Augmented reality in architecture: Rebuilding archeological heritage. International Archives of the Photogrammetry, Remote Sensing and Spatial Information Sciences - ISPRS Archives, 42(2W3), 311-315. https://doi.org/10.5194/isprs-archives-XLII-2-W3-311-2017

Dunleavy, M., \& Dede, C. (2014). Augmented reality teaching and learning. In Handbook of Research on Educational Communications and Technology: Fourth Edition (pp. 735-745). Springer New York. https://doi.org/10.1007/9781-4614-3185-5_59

Freitas, S. De. (2018). Are Games Effective Learning Tools? A Review of Educational Games The Review: A recent history of game science. 21, 7484.

Gede, I. D., Dhiyatmika, W., Gede, I. K., Putra, D., Made, N., \& Marini, I. (2015). Aplikasi Augmented Reality Magic Book Pengenalan Binatang Untuk Siswa Tk, 6(2), 120-127. https://doi. org/10.24843/LKJITI.6.2.16708

Habig, S. (2020). Who can benefit from augmented reality in chemistry? Sex differences in solving stereochemistry problems using augmented reality. British Journal of Educational Technology, 51(3), 629-644. https://doi. org/10.1111/bjet.12891

Irwansyah, F. S., Yusuf, Y. M., Farida, I., \& Ramdhani, M. A. (2018). Augmented Reality (AR) Technology on the Android Operating System in Chemistry Learning. IOP Conference Series:
Materials Science and Engineering, 288(1), 0-7. ht tps://doi.org/10.1088/1757-89 9X/288/1/012068

Khan, A., Egbue, O., Palkie, B., \& Madden, J. (2017). Active learning: Engaging students to maximize learning in an online course. Electronic Journal of E-Learning, 15(2), 107-115.

Khan, T., Johnston, K., \& Ophoff, J. (2019). The Impact of an Augmented Reality Application on Learning Motivation of Students. Advances in Human-Computer Interaction, 2019. https://doi. org/10.1155/2019/7208494

Kilgus, T., Heim, E., Haase, S., Prüfer, S., Müller, M., Seitel, A., Fangerau, M., Wiebe, T., Iszatt, J., Schlemmer, H. P., Hornegger, J., Yen, K., \& MaierHein, L. (2015). Mobile markerless augmented reality and its application in forensic medicine. International Journal of Computer Assisted Radiology and Surgery, 10(5), 573-586. https://doi. org/10.1007/s11548-014-1106-9

Laine, T. H., Nygren, E., Dirin, A., \& Suk, H. J. (2016). Science Spots AR: a platform for science learning games with augmented reality. Educational Technology Research and Development, 64(3), 507-531. https://doi.org/10.1007/s11423015-9419-0

Lester, J. C., Spires, H. A., Nietfeld, J. L., Minogue, J., Mott, B. W., \& Lobene, E. V. (2014). Designing game-based learning environments for elementary science education: A narrative-centered learning perspective. Information Sciences, 264, 4-18. https://doi.org/10.1016/j.ins.2013.09.005

Li, W., Nee, A. Y. C., \& Ong, S. K. (2017). A state-of-the-art review of augmented reality in engineering analysis and simulation. Multimodal Technologies and Interaction, 1(3). https://doi. org/10.3390/mti1030017

Lindgren, R., Tscholl, M., Wang, S., Johnson, E., \& Wang, S. (2016). Enhancing Learning and Engagement through Embodied Interaction within a Mixed Reality. Computers \& Education. https:// doi.org/10.1016/j.compedu.2016.01.001

Liu, Y., Holden, D., \& Zheng, D. (2016). Analyzing students' Language Learning Experience in an Augmented Reality Mobile Game: An Exploration of an Emergent Learning Environment. Procedia - Social and Behavioral Sciences, 
228(June), 369-374. https://doi.org/10.1016/j. sbspro.2016.07.055

Lucardie, D. (2014). The Impact of Fun and Enjoyment on Adult's Learning. Procedia - Social and Behavioral Sciences, 142, 439-446.

https://doi.org/10.1016/j.sbspro.2014.07.696

Marín, V., \& Sampedro-Rquena, B. E. (2019). La Realidad Aumentada en Educación Primaria desde la visión de los estudiantes. Alteridad, 15(1), 61-73.

https://doi.org/10.17163/alt.v15n1.2020.05

Martínez, N. M. M., Olivencia, J. J. L., \& Meneses, E. L. (2017). La realidad aumentada como tecnología emergente para la innovación educativa. Notandum, 125-140. https://doi.org/10.4025/notandum.44.11

Moloney, J., Globa, A., Wang, R., \& Roetzel, A. (2017). Serious games for integral sustainable design : Level 1. Procedia Engineering, 180, 17441753.

https://doi.org/10.1016/j.proeng.2017.04.337

Mossel, A., Schönauer, C., Gerstweiler, G., \& Kaufmann, H. (2012). ARTiFICe \pm Augmented Reality Framework for Distributed Collaboration. 11(3), 1-7. https://doi.org/10.20870/ IJVR.2012.11.3.2845

Nagalingam, V., \& Ibrahim, R. (2015). User Experience of Educational Games: A Review of the Elements. Procedia - Procedia Computer Science, 72, 423-433.

https://doi.org/10.1016/j.procs.2015.12.123

Nechypurenko, P. P., Starova, T. V., Selivanova, T. V., Tomilina, A. O., \& Uchitel, A. D. (2018). Use of augmented reality in chemistry education. CEUR Workshop Proceedings, 2257, 15-23. https://doi.org/10.31812/pedag.v51i0.3650

Nincarean, D., Bilal, M., Dayana, N., Halim, A., \& Abdul, H. (2013). Mobile Augmented Reality : the potential for education. 103, 657-664.

https://doi.org/10.1016/j.sbspro.2013.10.385

Oviatt, S., Arthur, A., \& Cohen, J. (n.d.). Quiet Interfaces that Help Students Think.

Potkonjak, V., Gardner, M., Callaghan, V., Mattila, P., Guetl, C., Petrović, V. M., \& Jovanović, K. (2016). Virtual laboratories for education in science, technology, and engineering: A review. Computers and Education, 95, 309-327. https:// doi.org/10.1016/j.compedu.2016.02.002

Prendes, C. (2015). Experiencias Prácticas Augmented Reality and Education: Analysis of. Pixel-Bit. Revista de Medios y Educación, (46), 187-203.

Rambli, D. R. A., Matcha, W., \& Sulaiman, S. (2013). Fun learning with AR alphabet book for preschool children. Procedia Computer Science, 25, 211-219. https://doi.org/10.1016/j. procs.2013.11.026

Rauschnabel, P. A., Felix, R., \& Hinsch, C. (2019). Augmented reality marketing: How mobile ARapps can improve brands through inspiration. Journal of Retailing and Consumer Services, 49(November 2018), 43-53.

https://doi.org/10.1016/j.jretconser.2019.03.004

Ruiz-ariza, A., Casuso, R. A., Suarez-manzano, S., \& Emilio, J. (2017). Effect of augmented reality game Pokemon GO on cognitive performance and emotional intelligence in adolescent young. Computers \& Education.

https://doi.org/10.1016/j.compedu.2017.09.002

Salinas, P., González-Mendívil, E., Quintero, E., Ríos, H., Ramírez, H., \& Morales, S. (2013). The development of a didactic prototype for the learning of mathematics through augmented reality. Procedia Computer Science, 25(81), 62-70. https://doi.org/10.1016/j.procs.2013.11.008

Sampaio, D., \& Almeida, P. (2016). Pedagogical Strategies for the Integration of Augmented Reality in ICT Teaching and Learning Processes. Procedia Computer Science, 100, 894-899. https://doi.org/10.1016/j.procs.2016.09.240

Scholz, J., \& Duffy, K. (2018). We ARe at home: How augmented reality reshapes mobile marketing and consumer-brand relationships. Journal of Retailing and Consumer Services, 44(May), 11-23.

https://doi.org/10.1016/j.jretconser.2018.05.004 Siwawetkul, W., \& Koraneekij, P. (2018). Effect of $5 \mathrm{E}$ instructional model on mobile technology to enhance reasoning ability of lower primary school students. Kasetsart Journal of Social Sciences, 41, 40-45.

https://doi.org/10.1016/j.kjss.2018.02.005

Spector, J. M., Merrill, M. D., Elen, J., \& Bishop, M. J. (2014). Handbook of research on educa- 
tional communications and technology: Fourth edition. Handbook of Research on Educational Communications and Technology: Fourth Edition, 1-1005. https://doi.org/10.1007/978-14614-3185-5

Steinkuehler, C., \& Squire, K. (2014). Videogames and learning. The Cambridge Handbook of the Learning Sciences, Second Edition, 377-394. https://doi.org/10.1017/CBO9781139519526.023 Streitz, N., \& Markopoulos, P. (2017). Distributed, ambient and pervasive interactions: 5 th international conference, DAPI 2017 held as part of HCI international 2017 Vancouver, BC, Canada, July 9-14, 2017 proceedings. Lecture Notes in Computer Science (Including Subseries Lecture Notes in Artificial Intelligence and Lecture Notes in Bioinformatics), 10291 LNCS, 612-626. 697-7 https://doi.org/10.1007/978-3-319-58697-7

Toledo Morales, P., \& Sánchez García, J. (2017). RealidadAumentada en Educación Primaria:efectos sobre el aprendizaje. RELATEC: Revista Latinoamericana de Tecnología Educativa, 16(1), 7992. https://doi.org/10.17398/1695-288X.16.1.79 https://doi.org/10.17398/1695-288X.16.1.79

Tomi, A. Bin, \& Rambli, D. R. A. (2013). An interactive mobile augmented reality magical playbook: Learning number with the thirsty crow. Procedia Computer Science, 25, 123-130. https:// doi.org/10.1016/j.procs.2013.11.015

Vázquez-Cano, E., Marín-Díaz, V., Oyarvide, W. R. V., \& López-Meneses, E. (2020). Use of augmented reality to improve specific and transversal competencies in students. International Journal of Learning, Teaching and Educational Research, 19(8), 393-408.

https://doi.org/10.26803/ijlter.19.8.21

Wedel, M., Bigné, E., \& Zhang, J. (2020). Virtual and augmented reality: Advancing research in consumer marketing. International Journal of Research in Marketing, 37(3), 443-465. https:// doi.org/10.1016/j.ijresmar.2020.04.004

Wr, D., Gxfdwlrq, L. Q., Uho, V., Rq, L. Q. J., Phwkrgrorjlhv, G., \& Dfwlylwlhv, D. Q. G. (2017). 07943075. April, 1683-1688.

Wu, H., Lee, S. W., Chang, H., \& Liang, J. (2013). Computers \& Education Current status, opportunities and challenges of augmented reality in education. Computers \& Education, 62, 41-49. https://doi.org/10.1016/j.compedu.2012.10.024 Wu, Y., Wu, Y., \& Yu, S. (2016). An Augmented-Reality Interactive Card Game for Teaching Elementary School Students. 10(1), 37-41.

Yang, S., Mei, B., \& Yue, X. (2018). Mobile Augmented Reality Assisted Chemical Education: Insights from Elements 4D. https://doi. org/10.1021/acs.jchemed.8b00017 